\title{
Electro-optic sampling at 90 degree interaction geometry for time-of-arrival stamping of ultrafast relativistic electron diffraction
}

\author{
C. M. Scoby, P. Musumeci, ${ }^{*}$ J. T. Moody, and M. S. Gutierrez \\ Department of Physics and Astronomy, UCLA, Los Angeles, California 90095, USA
}

(Received 29 September 2009; published 16 February 2010)

\begin{abstract}
In this paper we study a new geometry setup for electro-optic sampling (EOS) where the electron beam runs parallel to the $\langle 110\rangle$ face of a ZnTe crystal and the probe laser is perpendicular to it and to the beam path. The simple setup is used to encode the time-of-arrival information of a $3.5 \mathrm{MeV}<10 \mathrm{pC}$ electron bunch on the spatial profile of the laser pulse. The electric field lines inside the dielectric bend at an angle due to a relatively large $(n \sim 3)$ index of refraction of the ZnTe crystal. We found theoretically and experimentally that the EOS signal can be maximized with a proper choice of incoming laser polarization angle. We achieved single-shot nondestructive measurement of the relative time of arrival between the pump and the probe beams thus improving the temporal resolution of ultrafast relativistic electron diffraction experiments.
\end{abstract}

DOI: 10.1103/PhysRevSTAB.13.022801

PACS numbers: 42.65.Re, 07.78.+s, 41.75.Ht

\section{INTRODUCTION}

Electro-optic sampling (EOS) based temporal diagnostics of relativistic electron beams has been a very active field in the past decade. Favored by the spread of ultrafast laser techniques to the particle accelerator field, sub-ps relativistic electron beams are becoming increasingly common with applications in high gain free-electron lasers [1], in the context of laser based advanced accelerators [2], and more recently in direct structural dynamics measurements as ultrafast diffraction probes [3,4].

EOS techniques have been successfully applied to directly measure the beam near-field and its longitudinal profile $[5,6]$, to characterize beam generated radiation like coherent transition radiation [7], and to map the wakefields trailing the beam [8]. In all of these cases EOS naturally provides information on the time-of-arrival (TOA) difference between the laser and the electrons.

The application of EOS to the TOA measurement is very important for pump-probe experiments that require determining exactly how long after the laser excitation the probe beam (either an electron bunch or an x-ray pulse synchronized with it) captures the structure of the sample under study. Because of the nondestructive and single-shot nature of the EOS diagnostic, this scheme is ideal to provide a real-time direct measurement of such a delay.

For rf-based particle accelerators, the jitter on the beam TOA is usually linked to the phase jitter in the rf amplification chain and typically amounts to a few tenths of a degree of the rf frequency. For rf photoinjectors, the synchronization accuracy between the optical and rf low level oscillators adds another contribution to the total jitter. Furthermore, for low energy beams, rf amplitude fluctuations carry a significant contribution to the arrival time of

*musumeci@physics.ucla.edu the electron beam relative to an external laser pulse. The combination of these effects limits the temporal resolution of pump-probe techniques to a few hundred femtoseconds when using an S-band rf photoinjector. A possible solution to increase the temporal resolution in ultrafast dynamics studies is to time stamp each shot and then perform an offline temporal rearrangement of the data. A similar setup was proposed and successfully tested at the short pulse photon source beam line at SLAC [9], where a one-to-one correlation between the EOS time-of-arrival stamp and the evolution of an ultrafast phase transformation (the nonthermal melting of an InSb crystal [10]) was obtained.

We apply the EOS time-of-arrival diagnostics in the different context of relativistic ultrafast electron diffraction (UED) where the electrons are used directly as probes of atomic structural rearrangements on ultrafast time scales. The experiment takes place at the UCLA Pegasus photoinjector laboratory. The beam experimental parameters are particularly challenging, since the particles are not fully relativistic, and the charge per bunch is relatively low. To our knowledge, this is the first time EOS has been applied to the study of beams having $<4 \mathrm{MeV}$ energy and $<20 \mathrm{pC}$ beam charge.

A novel $90^{\circ}$ crossing spatial encoding geometry has been implemented for this measurement. Initially proposed by Srinisavan-Rao et al. [11], this scheme uses an electron beam propagating parallel to the crystal face and a laser pulse crossing the beam path perpendicularly. The experimental setup is relatively simple. Compared to other spatially encoding EOS techniques [9], the 90 degrees crossing EOS geometry requires no in-vacuum laser mirrors and uses a simple standard six-way cross on the beam line [12], thus minimizing the impedance seen by the beam. Further, it allows monitoring of the arrival time over a longer temporal window ( $>10 \mathrm{ps}$ ). Even though a variety of effects contribute to the smearing of the signal 
and make difficult the use of this scheme as longitudinal bunch profile monitor [13], the relative TOA can be determined with good (sub-100 fs) accuracy. The analysis of the interaction in this geometry is more complex than the standard case where the laser propagates collinearly with the electron beam and the crystal is oriented perpendicular to both. A careful analysis shows that, due to the large index of refraction in the dielectric, the laser probes bent field lines inside the medium. This implies, as we experimentally verified, that a preferred laser polarization angle exists to maximize the EOS signal intensity.

\section{TIME-STAMPING RELATIVISTIC ELECTRON DIFFRACTION PATTERNS}

The use of relativistic electrons has been proposed as a possible solution to improve the time resolution of UED [14]. Conventional UED setups employ 30-60 keV electrons and suffer greatly from bunch lengthening due to space charge effects $[15,16]$. In order to maintain sub-ps time resolution, the current paradigm is to employ only few thousands particles per bunch and obtain diffraction patterns by integrating over multiple pulses. At the UCLA Pegasus laboratory we have shown that, by increasing the beam energy to $3.5 \mathrm{MeV}$, we can generate electron beams with rms bunch lengths of few hundred fs and with up to 3 orders of magnitude more particles than currently available with nonrelativistic sources. These ultrashort electron pulses are suitable to obtain single-shot diffraction patterns as shown in Fig. 1, opening new possibilities in the study of ultrafast dynamics at the atomic scale. For the details of the ultrafast relativistic electron diffraction setup at the Pegasus laboratory, we refer the reader to other publications on the subject [4]. Here we quickly summarize in Table I the electron beam parameters which are relevant to the EOS measurements discussed in this paper.

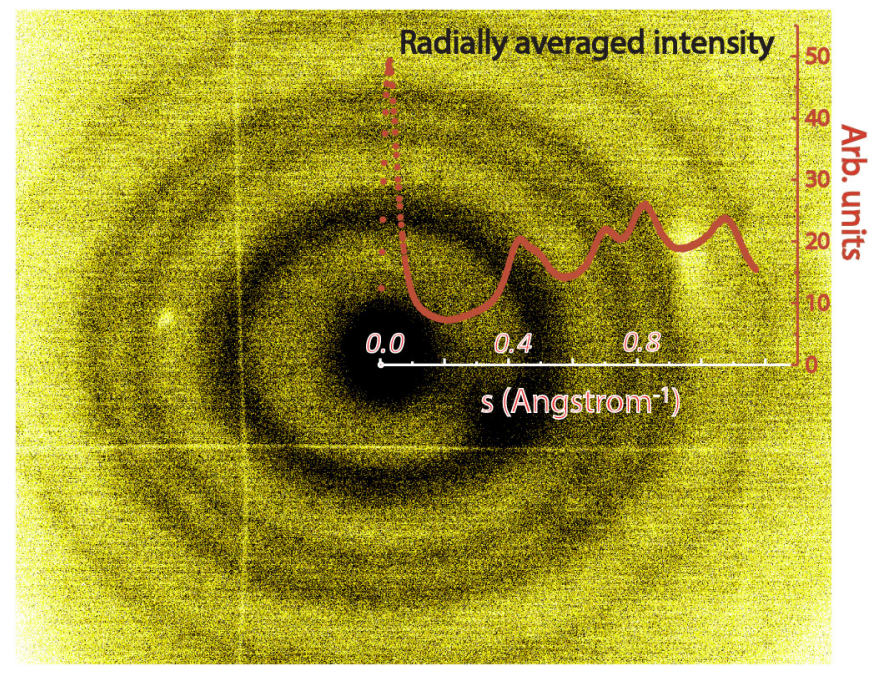

FIG. 1. (Color) Single-shot diffraction pattern taken with relativistic ultrafast electron diffraction. The radially averaged intensity profile is also shown.
TABLE I. Pegasus photoinjector parameters for electro-optic based time stamping.

\begin{tabular}{lc}
\hline \hline Beam energy & $3.5 \mathrm{MeV}$ \\
\hline Peak field at the cathode & $80 \mathrm{MV} / \mathrm{m}$ \\
Injection phase & $25^{\circ}$ \\
Beam charge & $10 \mathrm{pC}$ \\
Laser spot size (rms) & $500 \mu \mathrm{m}$ \\
Bunch length & $300 \mathrm{fs}$ \\
EOS distance from cathode & $80 \mathrm{~cm}$ \\
Electron diffraction target distance from cathode & $90 \mathrm{~cm}$ \\
\hline \hline
\end{tabular}

The temporal resolution of an ultrafast electron diffraction study is given by the convolution of the pump and probe pulse length, the velocity mismatch (which for ultrathin metal foil targets is negligible), and the relative timeof-arrival uncertainty [17]. In order to fully take advantage of the intense ultrashort probe beams generated by the $\mathrm{rf}$ photoinjector, one has to solve the difficult problem of minimizing the jitter in the time of arrival, or obtain a nondestructive TOA measurement and postprocess the images using this information. The latter approach is discussed in this paper.

In our experiment we use the same ultrashort laser pulse to generate the electrons at the cathode (probe), for depositing a burst of energy in the diffraction target (pump), and for the timing diagnostics. We initially split the $3 \mathrm{~mJ} 50 \mathrm{fs}$ long infrared laser pulse coming out of the coherent legend elite titanium:sapphire laser system. We send one-half of the energy to the frequency triplication stage to obtain the UV pulse that is used to illuminate the cathode and generate the high brightness probing electron beam. The other half is routed through a delay line and split again with a $90 / 10$ beam splitter just before it pumps the sample. We use the low energy pulse (about 5\% of the initial energy) for the EOS measurement. Since the last splitting happens only $60 \mathrm{~cm}$ before the target, the relative TOA of the

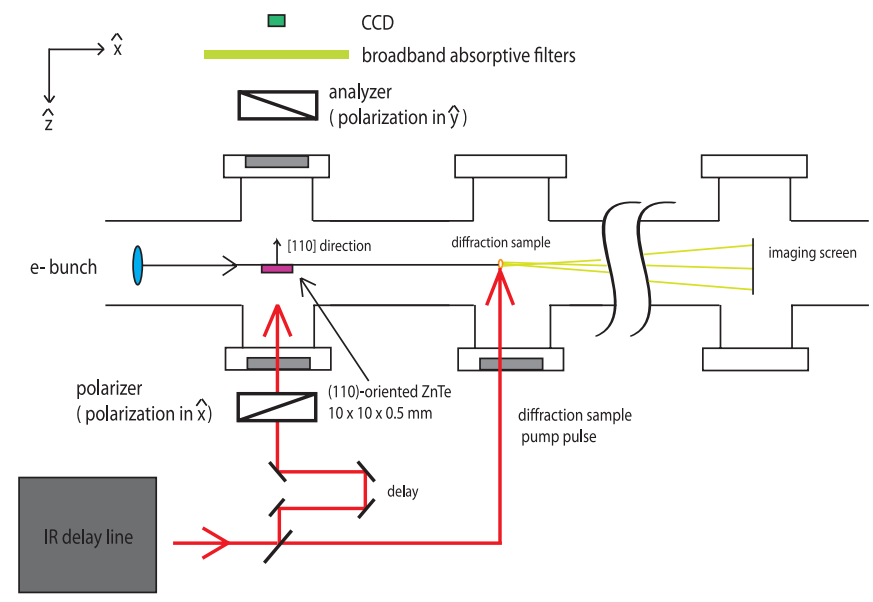

FIG. 2. (Color) Time-stamping scheme for ultrafast relativistic electron diffraction. 
electron beam with respect to the EOS laser pulse correlates perfectly with the delay between the pump and probe pulses at the diffraction target. Three mirrors are introduced as a delay so that the path lengths from the beam splitter to the EOS interaction point and from the beam splitter to the diffraction target are equal, accounting for the time that the electron beam takes to travel from one to the other (see Fig. 2).

\section{ELECTRO-OPTIC SAMPLING AT 90 DEGREES INTERACTION}

Single-shot EO bunch monitors can be broadly divided into different categories depending on the way one chooses to read out the signal: temporally encoding, spectrally encoding, and spatially encoding $[18,19]$. We adopted the simple spatially encoding 90 degrees crossing geometry proposed in Ref. [11] which has the advantage of avoiding temporal or spectral manipulations of the laser pulse. The ability to monitor a relatively long time window (thus eliminating the need to independently move the delay of the EOS and of the pump pulse while doing an UED time scan) and a very good temporal resolution are important advantages of this geometry for our application.

$\mathrm{A}\langle 110\rangle$-cut ZnTe crystal is aligned parallel to the direction of bunch propagation. The crystal is lowered until its bottom edge is $1.5 \mathrm{~mm}$ above the beam axis. The linearly polarized probe laser pulse then propagates along the direction normal to the crystal face. A half-wave plate is used to allow the rotation of the input laser polarization. A polarizer further ensures the uniformity of probe pulse polarization and reduces the background on the measurement. The laser then passes through the $10 \times 10 \times 0.5 \mathrm{~mm}$ $\mathrm{ZnTe}$ crystal, which acts as the electro-optically active medium (see Fig. 3). The pulse then enters an analyzer that is set $90^{\circ}$ from the input polarization axis to reject all except the modulated part of the probe pulse. The laser probe pulse finally impinges on a $640 \times 480$ pixel CCD, where we detect the transmitted intensity, $I_{\mathrm{tr}}$. Neutral density filters are used after the analyzer to maximize the dynamic range of the measurement and avoid saturation on the CCD. The CCD array employed in this experiment is a $1 / 4^{\prime \prime}$ diagonal and the laser beam (transported from the amplifier output without any lens) is $\sim 12 \mathrm{~mm}$ FWHM in size and hence overfills the aperture, making the laser intensity very uniform over the detector area.

The simplest explanation of the electro-optic effect is that it turns the crystal into a voltage-controlled wave plate [20]. The phase difference imposed on light traveling through a birefringent material takes the general form

$$
\Gamma=\frac{2 \pi}{\lambda} L \Delta n
$$

where $\lambda$ is the wavelength of the light, $L$ is the thickness of the material, and $\Delta n$ is the difference between the indices of refraction for the light polarization along the fast and slow axes of the birefringent crystal. The electro-optic effect (also called the Pockels effect) is characterized by a linear induced birefringence in response to an external electric field.

Restricting the discussion to a beam field $\vec{E}_{b}$ in the $\langle 110\rangle$ plane of the ZnTe crystal, the difference in refractive index due to the EO effect takes the form

$$
\Delta n=n_{0}^{3} r_{41}\left|\vec{E}_{b}\right| \frac{\sqrt{1+3 \sin ^{2} \varphi}}{2}
$$

where $n_{0}$ is the nominal refractive index at the wavelength of the input light, $r_{41}$ is the electro-optic coefficient, and $\varphi$ is the angle that $\vec{E}_{b}$ makes with the (001) axis of the ZnTe crystal. Higher order terms in $\left|\vec{E}_{b}\right|$ (e.g. the Kerr effect) have been ignored due to their small relative magnitudes.

The orientation of the induced optical axes (i.e. the angle $\Psi$ in Fig. 4) is related to the direction of $\vec{E}_{b}$ by the expression

$$
\tan 2 \Psi=-2 \tan \varphi
$$

If a laser is sent through a combination of a polarizer and an analyzer oriented at 90 degrees with respect to each other, the transmitted intensity $I_{\text {tr }}$ is nearly zero (zerooptical bias). Introducing the ZnTe crystal between the two crossed polarizers and turning the beam field on causes the linear polarization to pick up an elliptical polarization component. The analyzer will pick out the nonzero component of the rotated polarization, so that the transmitted intensity $I_{\text {tr }}$ effectively represents a two-dimensional snapshot of the birefringence induced in the crystal by the beam electric field. Application of the Jones matrix formalism [20] shows that the maximum transmitted intensity is obtained for an input laser polarization angle oriented at 45 degrees with respect to the induced optical axes.

The horizontal position of the signal is related to the TOA of the electron bunch with respect to the laser pulse. Using Fig. 3 as a reference, later (earlier) arrival of the laser shifts the signal horizontally to the left (right). A CCD camera is used to read out the time information of the bunch electric field, which is mapped onto the horizontal profile of the transmitted laser pulse.

In practice, a small fraction of the laser pulse leaks onto the CCD even without the beam field. Contributions to the total background are due to the finite extinction ratio of the polarizers and to the scattering and residual birefringence in the electro-optic crystal itself. Background images are acquired without the beam and subtracted from the images taken with the beam on. While a small optical bias has been found to maximize the EOS signal-to-noise ratio [21], in these first measurements we have chosen to work as closely as possible to the zero-optical bias to minimize the effect of amplitude and shape fluctuations of the laser.

A typical image of the 2D field profile is shown in Fig. 5. Here the laser timing is such that the crystal is illuminated when the electron beam has traveled nearly to the end of it. 
(d)

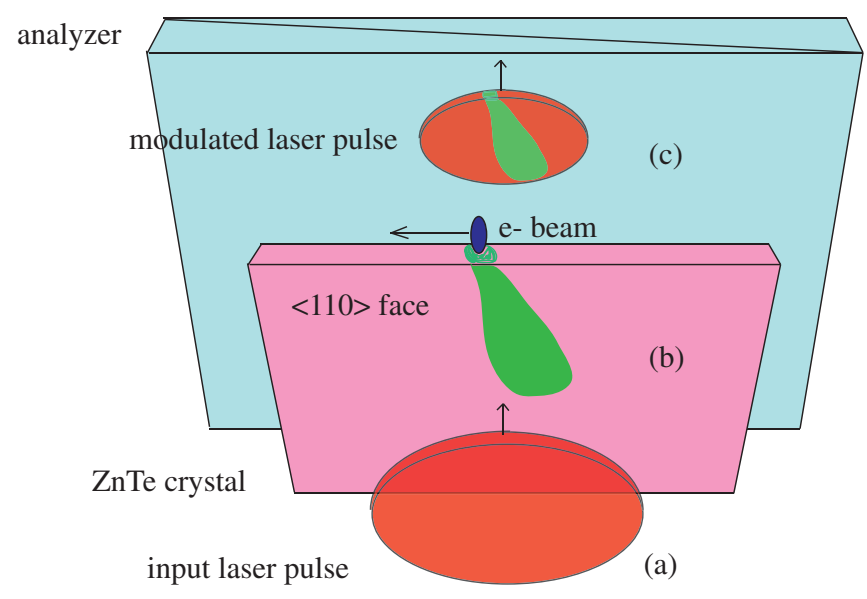

FIG. 3. (Color) Top-down/frontal view showing single-shot EOS 2D geometry. (a) A linearly polarized laser pulse approaches. The half-wave plate and the polarizer used to control the polarization direction are not shown. (b) An e- bunch induces birefringence in the ZnTe crystal. (c) The e- bunch profile is imprinted on the laser pulse as a phase modulation. (d) The analyzer removes all but the modulated part of laser, which is detected on the CCD.

By looking at the figure, it is immediately apparent that the electric field shows a clear angle with respect to the beam direction. In vacuum, the field of a nearly relativistic electron beam points perpendicularly to the beam direction. In our case, since the electron bunch length is sub-ps,

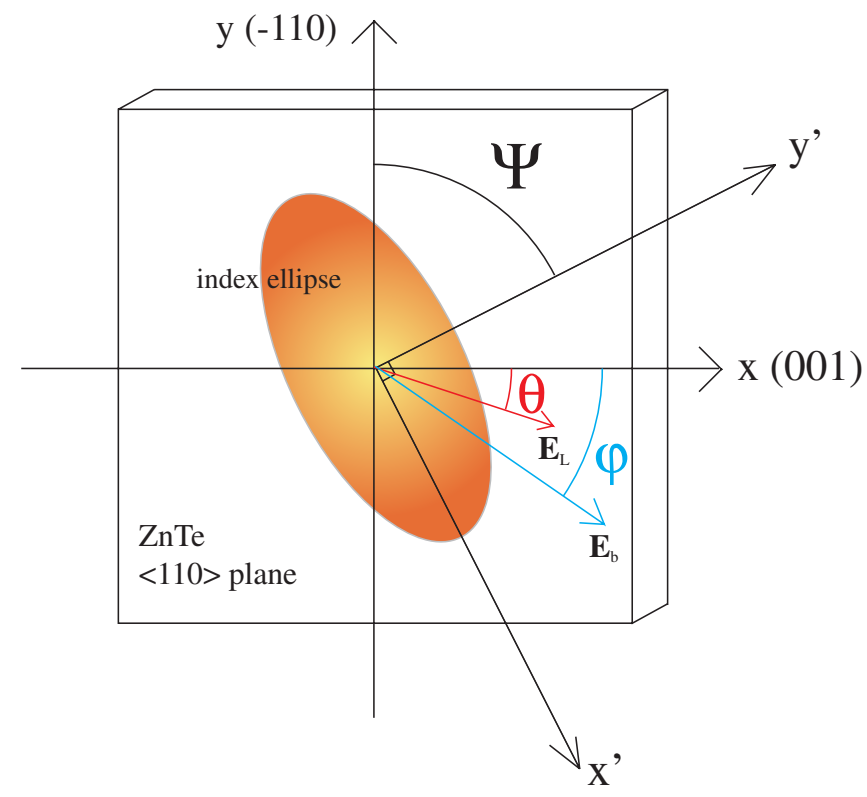

FIG. 4. (Color) Index ellipsoid projection onto the 110 face of the $\mathrm{ZnTe}$ crystal showing the angles of the beam electric field $\vec{E}_{b}$ and of the laser electric field $\vec{E}_{L}$. the frequencies associated with the beam field will be in the $\mathrm{THz}$ range. Because of the relatively large index of refraction of ZnTe $(n \sim 3)$ at the these frequencies, the electromagnetic field travels more slowly in the crystal than the beam does in vacuum. In an infinitely wide slab geometry, the angle of the field lines with respect to the beam direction would be exactly the one given by the Cherenkov condition $\varphi_{c}=\arcsin (1 / \beta n) \sim 19^{\circ}$. In absence of the dielectric, the electric field of the quasirelativistic beam would have a direction mostly perpendicular to the beam path. In our three-dimensional geometry, the field can be seen as a superposition of the two cases, with matching boundary conditions at the crystal surfaces. In other words, the field "leaks" around the thin crystal, and appears to move slightly faster. For this reason, the angle is larger than $\varphi_{c}$ [12]. It should also be noted that the laser useful region, taking into account the $\mathrm{CCD}$ and polarizer apertures, was 2 to 3 times smaller than the $10 \mathrm{~mm}$ crystal width. In order to avoid transient effects from the crystal boundary, where the field angle could be not well defined, the laser was aligned to illuminate the downstream side of the crystal (away from the cathode). From the image, the angle can be estimated to be $\varphi_{\text {image }} \sim 32^{\circ}$. Because of the large frequency content in the beam spectrum, both spatial and temporal dispersion contribute to the broadening of the horizontal profile and to the angular spread of the field lines as the electromagnetic pulse travels into the crystal. Experimentally, we also found that the angle of the field lines strongly depends on the beam steering both horizontally and vertically.

By rotating the two crossed polarizers by the same angle and adjusting the input half-wave plate to maintain constant laser illumination of the crystal, we can control the

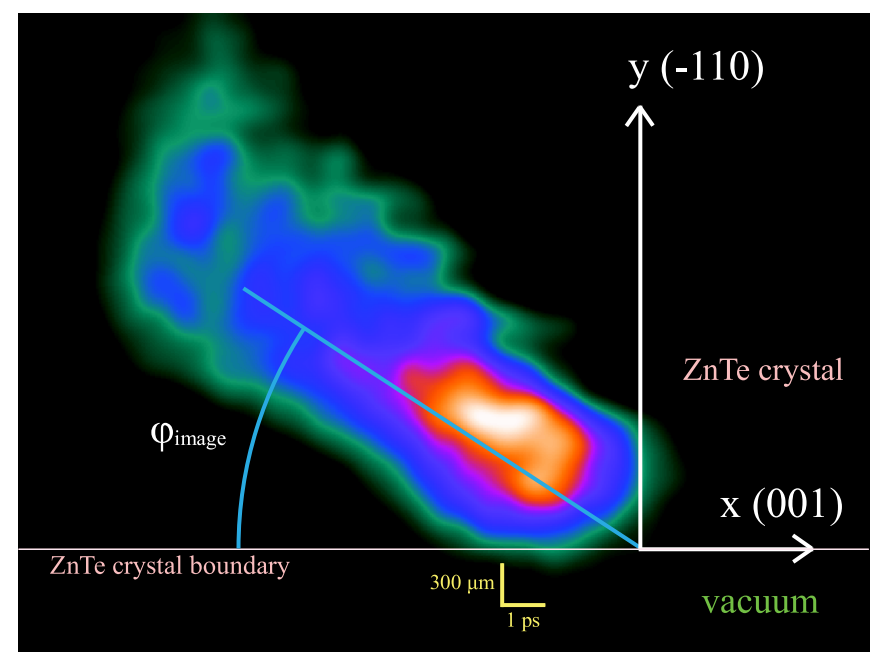

FIG. 5. (Color) A single frame taken from the CCD shows the background-subtracted modulated laser pulse. The temporal information is encoded onto the horizontal axis and the vertical axis is related to the propagation of the beam field in the material. 


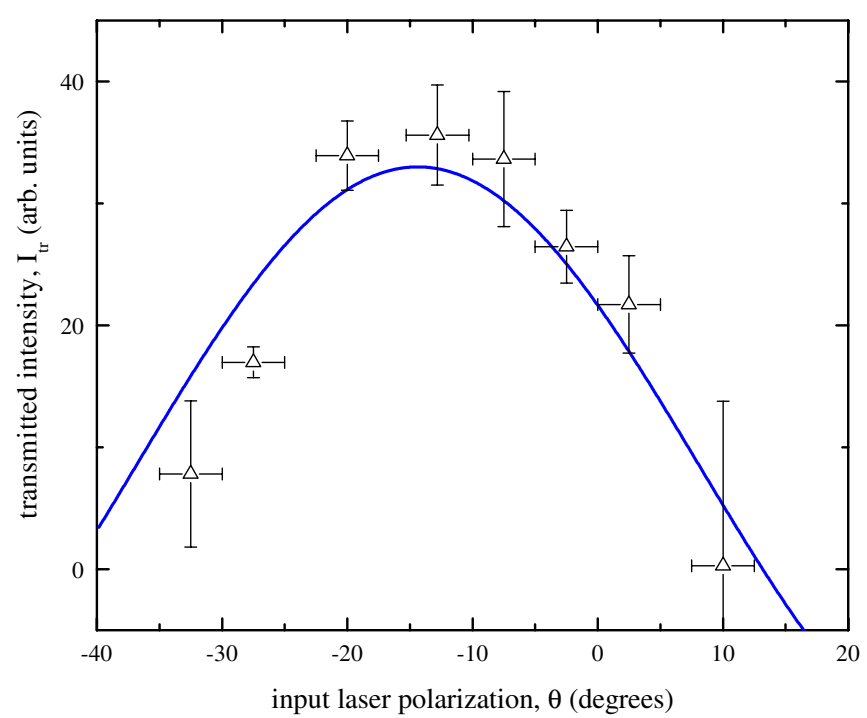

FIG. 6. (Color) EOS transmitted intensity as a function of input laser polarization angle. The maximum signal occurs at $\theta \sim$ $-17^{\circ}$. A $\sin ^{2}$ fit (solid line) is used to guide the eye.

input laser polarization angle $\theta$ (see Fig. 4) and study the amplitude of the electro-optic signal as a function of this quantity. The result is shown in Fig. 6. The data shows a maximum of the signal for $\theta=-17^{\circ}$. This indicates that the induced optical axes in the crystal are rotated by an angle $\Psi=\theta-\pi / 4$ from the horizontal. Using Eq. (3) we obtain $\varphi \sim 36^{\circ}$ for the direction of $\vec{E}_{b}$. This result is in agreement with the result inferred in the analysis of the $2 \mathrm{D}$ image when taking into account experimental errors. Optimization of the incoming laser polarization allows us to measure the electro-optic signal with very low charge beams.

\section{TIME-OF-ARRIVAL MEASUREMENT OF AN ULTRASHORT LOW CHARGE BEAM}

Various effects need to be taken into account when estimating the temporal resolution of our EOS technique for longitudinal bunch profile measurements. Since a horizontal lineout of the CCD image is a projection of the bunch field profile due to the bending of the field lines inside the dielectric, this must be accounted for in order to retrieve the exact bunch length. The low energy of the beam implies a relatively large opening angle of the field, which will spatially broaden the EOS signal as read on the CCD camera [22]. For a $1 \mathrm{~mm}$ distance between the beam axis and the crystal, the smear of the signal can be estimated as $2 \times 1 \mathrm{~mm} / c \gamma \cong 800 \mathrm{fs}$, where $\gamma \sim 8$ is the beam Lorentz relativistic factor. The thickness of the crystal, $0.5 \mathrm{~mm}$ in this experiment, which in simple terms has an effect equivalent to the shutter speed of the camera we are using to observe the beam, also limits the minimum bunch length that can be detected with this geometry to
$0.5 \mathrm{~mm} \cdot n / c \cong 3 \mathrm{ps}$, where $n$ is the index of refraction of the crystal at $800 \mathrm{~nm}$. Finally, ZnTe has an absorption band at $3 \mathrm{THz}$. The resulting dispersion of the $\mathrm{THz}$ components of the beam field in the crystal (both temporally and spatially) naturally limits the resolution to a few hundred femtoseconds.

In spite of the smearing introduced by these effects which are responsible for broadening the width of the signal from the ultrashort beam to $>2 \mathrm{ps}$, this technique works very well as a TOA diagnostic because the position of the bunch centroid is unaffected. For given settings of the rf photoinjector, the spreading in the EOS signal is fixed so that the position of the peak is a faithful indicator of the relative TOA of the laser with respect to the electron beam. In practice the TOA information is extracted by taking a horizontal lineout of the 2D image and fitting a peak to determine the beam position.

In order to obtain a calibration from pixel number to time, we recorded the position of the peak in the EOS signal as a function of the position of the infrared laser delay line. A simple linear fit yields a calibration of $28 \mathrm{fs} /$ pixel, which matches the estimate obtained using the CCD camera pixel size $(8.8 \mu \mathrm{m})$ and the relativistic beam velocity.

The accuracy of the single-shot time stamp from the EOS-based measurement is determined by how well the peak of the signal is measured. The $2 \mathrm{D}$ image profile is very reproducible, fluctuating mostly in the horizontal direction due to the TOA time jitter. The most important systematic error to the TOA determination comes from the beam pointing jitter which alters the spatial configuration of the field in the crystal thus appearing as a false timing error. In order to give an estimate for this effect, we have tried to quantify the shot-to-shot differences in the $2 \mathrm{D}$ images which contain the information of the field distribution in the crystal. By taking lineouts at different rows of the 2D image (and hence different heights from the beam axis in the crystal), one finds different peak positions due to the field angle. If the 2D images were perfectly reproducible, these values should correlate perfectly. By restricting the analysis to a region of the $2 \mathrm{D}$ image $<0.5 \mathrm{~mm}$ from the crystal edge, the mean rms deviation from the perfect correlation observed over 100 consecutive images is $50 \mathrm{fs}$, which can be taken as a self-consistent value for the accuracy of the TOA measurement from a single-shot EOS image.

The relative TOA jitter was measured by acquiring the EOS signal for 1000 consecutive shots. The measurement is taken with the feedback loops on the rf phase and $\mathrm{rf}$ amplitude closed in order to correct slow drifts over time scales longer than a few minutes. The TOA distribution is displayed in Fig. 7, which shows a standard deviation of $700 \mathrm{fs}$. This number is high compared to that of other stateof-the-art accelerator laboratories, and an upgrade to the shot-to-shot phase and amplitude stability of the rf system 


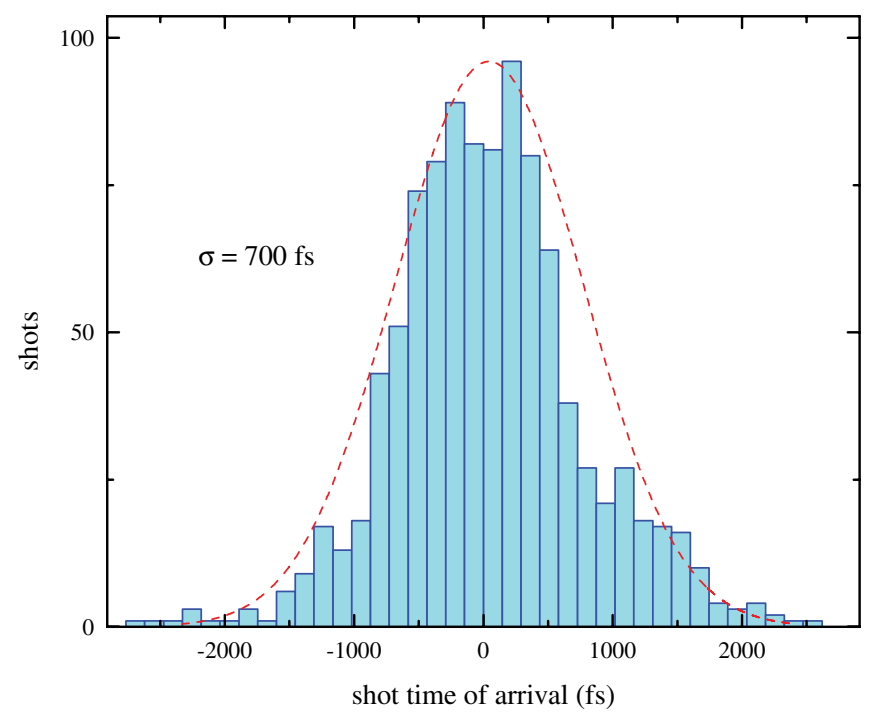

FIG. 7. (Color) Timing jitter histogram.

is planned at Pegasus to improve this parameter. Without the implementation of a nondestructive single-shot TOA measurement technique like the one described in this paper, the resolution of a pump-probe study by ultrafast relativistic electron diffraction would be limited by such jitter to $>700 \mathrm{fs}$. In our system, thanks to the described online TOA determination, the temporal resolution of an ultrafast electron diffraction study can be calculated as the quadrature sum of the electron bunch length (which for low beam charge is $<150 \mathrm{fs}$ ), the laser pulse length ( $<80 \mathrm{fs}$ ), and the accuracy of the EOS-based measurement and is better than $200 \mathrm{fs}$.

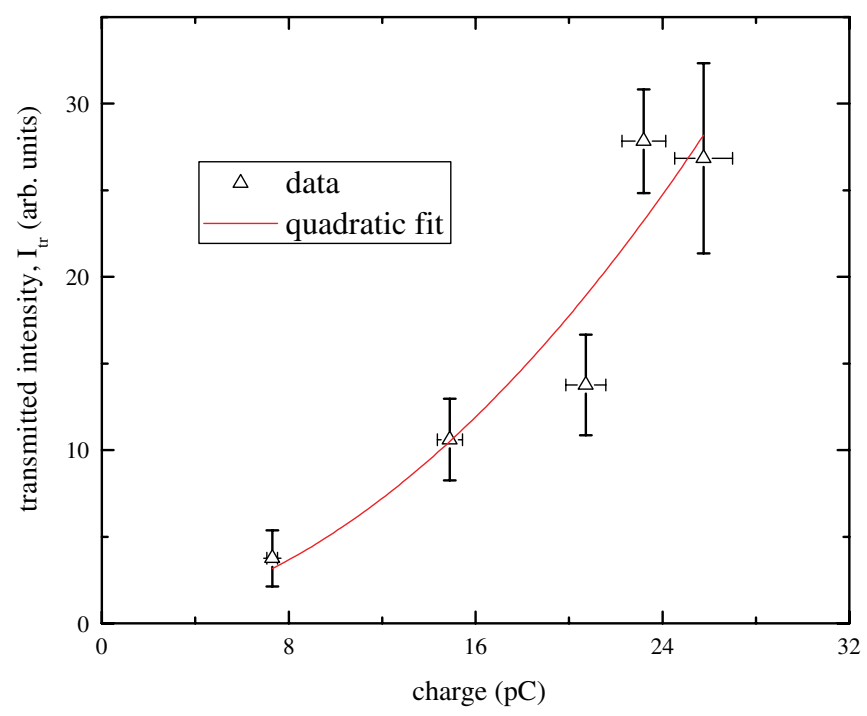

FIG. 8. (Color) EOS transmitted intensity as a function of electron bunch charge. The minimum charge for which an EOS signal was measured here is $7.3 \mathrm{pC}$. A quadratic fit is used to guide the eye.
Finally, we show in Fig. 8 the amplitude of the EOS signal as a function of charge. This confirms that the implemented setup is able to monitor beams of very low charges (i.e. $<10 \mathrm{pC}$ ) as required by our UED application. As expected, the amplitude of the signal is quadratic as a function of charge. In fact, combining Eqs. (1) and (2), it is easy to see that the phase difference induced by the electrooptic effect $\Gamma$ is linear in the applied electric field and the beam charge. For zero-optical biasing and a small induced birefringence, the intensity transmitted between the two crossed polarizers is $\propto \sin ^{2} \Gamma \cong \Gamma^{2}$.

Using the total incoming infrared laser intensity (measured without the analyzer) as a normalization for the electro-optic signal, we calculate for the $10 \mathrm{pC}$ case $\Gamma=$ $10 \mathrm{mrad}$. This corresponds to an electric field $E_{b} \cong$ $100 \mathrm{kV} / \mathrm{m}$ which matches well the field expected for a 3.5 MeV pancake beam at $1 \mathrm{~mm}$ from the beam axis. Such small modulation is at the lower limit of what can be detected using the 8-bit CCD employed in the experiment. Using a better CCD array, and decreasing the shot-to-shot fluctuations on the laser intensity, it could be possible to push this limit further down. Moreover, by improving the beam pointing jitter, one could use a larger part of the 2D image for TOA determination and obtain a signal even for lower charges. Assuming such improvements, since the ZnTe crystal thickness contributes more to the spreading of the peak than to its amplitude, a thinner crystal could be employed without suffering too much signal loss.

\section{CONCLUSION}

We have implemented a single-shot nondestructive time-of-arrival measurement for ultrashort high brightness beams suitable for ultrafast relativistic electron diffraction. The measurement technique is based on a novel electrooptic sampling scheme using a crossing geometry where the laser and the electron beam are traveling in perpendicular directions. Because of the relatively large index of refraction in the dielectric medium, the field lines in the crystal are bent. This requires a particular angle for the incoming laser polarization to maximize the signal.

For electron diffraction, we typically run with $<10 \mathrm{pC}$ at the cathode and apply a relatively weak focusing with the emittance compensation solenoid to collimate the beam and minimize the beam divergence. It was important to demonstrate the possibility of obtaining an EOS signal with these accelerator settings. Relative TOA between the laser and the electron beam has been measured for 1000 consecutive shots in these conditions, obtaining a jitter of $700 \mathrm{fs}$ rms. Though further steps should be taken to minimize the jitter, the temporal resolution of an electron diffraction ultrafast study would not be limited by this quantity due to the nondestructive nature of the diagnostic. Improvements to the EOS measurement technique by nonzero-bias optical schemes and extensions to the measurement of the longitudinal bunch profile are under study. 


\section{ACKNOWLEDGMENTS}

This work was supported by ONR-JTO Award No. N000140711174 and DOE Grant No. DE-FG0292ER40693.

[1] Y. Ding, A. Brachmann, F. J. Decker, D. Dowell, P. Emma, J. Frisch, S. Gilevich, G. Hays, Ph. Hering, Z. Huang, R. Iverson, H. Loos, A. Miahnahri, H. D. Nuhn, D. Ratner, J. Turner, J. Welch, W. White, and J. Wu, Phys. Rev. Lett. 102, 254801 (2009).

[2] W. P. Leemans, B. Nagler, A. J. Gonsalves, Cs. Tóth, K. Nakamura, C. G. R. Geddes, E. Esarey, C. B. Schroeder, and S. M. Hooker, Nature Phys. 2, 696 (2006).

[3] J. B. Hastings, F. M. Rudakov, D. H. Dowell, J. F. Schmerge, and J.D. Cardoza, Appl. Phys. Lett. 89, 184109 (2006).

[4] P. Musumeci, J. T. Moody, and C.M. Scoby, Ultramicroscopy 108, 1450 (2008).

[5] X. Yan, A.M. MacLeod, W. A. Gillespie, G. M. H. Knippels, D. Oepts, A.F.G. van der Meer, and W. Seidel, Phys. Rev. Lett. 85, 3404 (2000).

[6] G. Berden, W. A. Gillespie, S. P. Jamison, E. A. Knabbe, A. M. MacLeod, A. F. G. van der Meer, P. J. Phillips, H. Schlarb, B. Schmidt, P. Schmser, and B. Steffen, Phys. Rev. Lett. 99, 164801 (2007).

[7] J. Van Tilborg, C. B. Schroeder, C. V. Filip, Cs. Tóth, C. G. R. Geddes, G. Fubiani, R. Huber, R. A. Kaindl, E. Esarey, and W. P. Leemans, Phys. Rev. Lett. 96, 014801 (2006).

[8] M. J. Fitch, A.C. Melissinos, P.L. Colestock, J. P. Carneiro, H. T. Edwards, and W. H. Hartung, Phys. Rev.
Lett. 87, 034801 (2001).

[9] A. L. Cavalieri et al., Phys. Rev. Lett. 94, 114801 (2005).

[10] A. Rousse et al., Nature (London) 410, 65 (2001).

[11] T. Srinivasan-Rao, M. Amin, V. Castillo, D. M. Lazarus, D. Nikas, C. Ozben, Y. K. Semertzidis, A. Stillman, and T. Tsang, Phys. Rev. ST Accel. Beams 5, 042801 (2002).

[12] C. M. Scoby et al., in Proceedings of 2009 PAC Conference, Vancouver, 2009 (to be published); in Proceedings of the Thirteenth Advanced Accelerator Concepts Workshop, Santa Cruz, 2008, AIP Conf. Proc. No. 1086 (AIP, New York, 2009), p. 655.

[13] X. Yang, T. Tsang, T. Rao, J. B. Murphy, Y. Shen, and X. J. Wang, Appl. Phys. Lett. 95, 231106 (2009).

[14] X. J. Wang, Z. Wu, and H. Ihee, in Proceedings of the 20th Particle Accelerator Conference, Portland, OR, 2003 (IEEE, New York, 2003).

[15] A. H. Zewail, Annu. Rev. Phys. Chem. 57, 65 (2006).

[16] J.R. Dwyer et al., Phil. Trans. R. Soc. A 364, 741 (2006).

[17] X. J. Wang, D. Xiang, T. J. Kim, and H. Ihee, J. Korean Phys. Soc. 48, 390 (2006).

[18] I. Wilke, A. M. MacLeod, W. A. Gillespie, G. Berden, G. M. H. Knippels, and A. F. G. van der Meer, Phys. Rev. Lett. 88, 124801 (2002).

[19] G. Berden, S. P. Jamison, A. M. MacLeod, W. A. Gillespie, B. Redlich, and A. F. G. van der Meer, Phys. Rev. Lett. 93, 114802 (2004).

[20] J. Van Tilborg, Ph.D. thesis, Technische Universiteit Eindhove, 2005.

[21] Y. W. Parc, J. Y. Huang, and C. Kim, Proceedings of 2007 FEL Conference, Novosibirsk, Russia, 2007, p. 103.

[22] Y. W. Parc, J. Hong, I. S. Ko, C. Kim, J. Y. Huang, and S. H. Lee, Jpn. J. Appl. Phys. 47, 342 (2008). 